\title{
Correction to: Next Generation Sequencing (NGS) Application in Multiparameter Gene Expression Analysis
}

\section{Degeng Wang and Andrey L. Karamyshev}

\section{Correction to:}

Chapter 2 in: Phouthone Keohavong et al. (eds.), Molecular Toxicology Protocols, Methods in Molecular Biology, vol. 2102, https://doi.org/10.1007/978-1-0716-0223-2_2

1. Chapter 2 was inadvertently published with the contributing author's name printed as Andrey L. Karamychev, whereas it should have been Andrey L. Karamyshev. This correction has been updated in the book.

2. The name of the university in the affiliation section was incorrectly printed as "Texas Tech University Health Science Center". It has now been corrected as "Texas Tech University Health Sciences Center".

The updated online version of this chapter can be found at https://doi.org/10.1007/978-1-0716-0223-2_2. 